\title{
Model Comparison: a Systematic Mapping Study
}

\author{
Lucian José Gonçales, Kleinner Farias, Murillo \\ Scholl, Maurício Veronez \\ PIPCA, University of Vale do Rio dos Sinos (Unisinos) \\ São Leopoldo, RS, Brazil \\ lucianjosegoncales@gmail.com, \\ kleinnerfarias@gmail.com,murillosholl@hotmail.com, \\ veronez@unisinos.br
}

\author{
Toacy Oliveira \\ PESC/COPPE, Federal University of Rio de Janeiro \\ (UFRJ) \\ Rio de Janeiro, RJ, Brazil \\ toacy@cos.ufrj.br
}

\begin{abstract}
Context: Model comparison plays a central role in many software engineering activities. However, a comprehensive understanding about the state-of-art is still required. Goal: This paper, therefore, aims at classifying, identifying publication fora, and performing thematic analysis of the current literature in model comparison for creating an extensive and detailed understanding about this area, thereby determining gaps by graphing and pinpointing in which research areas and for which study types a shortage of publications still exits. Method: We have conducted a systematic mapping study to scrutinize those contributions produced over time, which research topics have most investigated, and which research methods that have been applied. For this, we have followed well-established empirical guidelines to define and apply a systematic mapping study. Results: The results are: (1) majority of studies (14 out of 40) provide generic model comparison techniques, rather than comparison techniques for UML diagrams; (2) a categorization and quantification of the current studies in a variety of dimensions; and (3) an overview of current research topics and trends.
\end{abstract}

Keywords-component; model comparison, model matching, mapping study, model similarity.

\section{INTRODUCTION}

Model Driven-Engineering (MDE) is a model-centric approach where developers focus on elaborating, maintaining, and evolving design models in different levels [1][2]. In this context, model comparison plays a central role in many MDE activities as software models are in constant changes i.e., deletions, additions, and updates, are frequently occurring in such artifacts [2][3]. The goal behind managing models is to abstract software development process, i.e., guided by ideas and stakeholders goals instead typing a thousand of line codes. Academia has provided comparison techniques; at the same time, industry has provided robust tools. However, the resolution of model comparison did not reach an ideal scenario. Robust tools like IBM RSA [4], Epsilon [5] and MATA [6], still suffer from model comparison problems.

This is due their functionalities are far from providing a precise and large-scale computation in synchronizing and matching models. In [7][8], comparison problem is usually associated to graph isomorphism, well-know to be hard to resolve. Then, we can conjecture that current tools and comparison techniques do not solve the entire comparison problem yet, and then we still stand on the craftsmanship era.
We understand that a comprehensive understanding about the state-of-the-art is crucial for evolving the current comparison techniques.

This paper, therefore, aims at classifying, identifying publication fora, and performing thematic analysis of the current literature in model comparison for creating an extensive and detailed understanding of the state-of-the-art in this area. Moreover, we seek to determine gaps by graphing and pinpointing in which research areas and for which study types a shortage of publications still exits.

In this sense, we have conducted a systematic mapping study [9][10] to (1) scrutinize those contributions produced over time, and (2) characterize previously published model comparison approaches, i.e., which research topics have been most investigated, and which research methods that have been applied. For this, we have followed well-established empirical guidelines for defining and applying systematic mapping study (e.g., [10][11][12][13][14][15]). This method focuses on collecting statistical data related to a set of research questions, and provides a body of knowledge to future researches.

Our results show that approximately $34 \%$ of all studies (14 out of 40) provide generic model comparison techniques, rather than comparison techniques for UML diagrams [16]. Moreover, we categorize and quantify the current studies in a variety of dimensions, and give an overview of current research topics and trends. Finally, we have observed that existing literature has focused on providing repeated solutions for similar problems rather than on innovative approaches.

The remainder of this paper is organized as follows. Section II discusses the related work. Section III presents the SMS planning, i.e., the main steps for guiding the Systematic Mapping Study (SMS). Section IV presents the study results. Section V discusses the results by presenting a study map. Section VI presents some complementary results. Section VII shows the threats to validity. Finally, Section VIII presents some conclusions.

\section{RELATED WORK}

This section reports a series of studies, including surveys, mapping studies, and systematic reviews, which have previously reviewed the state-of-the-art in model comparison. To the best of our knowledge, this paper is the first to investigate the main research question proposed in Table 1. 
There is a lack in the academia and industry for systematic and mapping studies that summarize model matching approaches. That is, there is no study showing a widespread view about model composition techniques or even providing a body of knowledge to future researches. In [17], authors present an analysis about model comparison techniques showing its differences and trade-offs according the matching scenario, and in [18], authors present a survey about the stateof-the-art comparison approaches where they categorize approaches by type of models. Therefore, both studies do not focus in present a summary of studies through mapping study protocols.

Usually, current surveys indirectly address model comparison in the context of clone detection and model versioning, rather than systematically deal with comparison issues in the field of UML model composition, for example. In [19] the authors present a survey about tools that executes three-way merge inside the versioning control system. Still, they hightligth that comparison task is essential to update models in repository. Alanen and Porres [20] present a description of three model-independent differentiation algorithms in the field of model versioning.

Given that model comparison is widely used, many approaches have been proposed, including UML and non-UML based ones. In [21], Salami and Ahmed describe the state-ofthe-art works considering reuse of UML artifacts. Nevertheless, they cover only UML approaches. Selonen [22] presents a survey on model comparison approaches focused on UML models. Unfortunately, none of them provides a careful report classifying, identifying publication fora, and performing thematic analysis of the current literature in model comparison, hampering the creation of an extensive and detailed understanding about this area.

To sum up, there has been very limited empirical research reporting and characterizing the state-of-the-art of model comparison. More specifically, we have identified five key gaps in the current literature: (1) a lack of understanding about how model comparison has been investigated in the last years, and on which perspectives it has been done; (2) a gap to draw a "big picture" view of model comparison beyond UML, such as the degree of abstraction of comparison mechanisms; (3) there is no understanding as to what extent the comparison techniques are accurate, and which research methods have been used to investigate such techniques; (4) limited knowledge about which diagrams are supported by the comparison techniques, and which improvement points are more urged; and, finally, (5) an overview about how automated the model comparison techniques are.

\section{SMS PLAN STUDY}

This section describes the scope and essential steps for executing the systematic mapping study (SMS). Section III.A summarizes the researcher questions. Section III.B defines the strategy for the searching studies. Section III.C lists the inclusion and exclusion criteria for studies selection. Finally, Section III.D specifies the data extracted from selected studies.

\section{A. Research Questions}

Table I shows the research questions addressed in this study and their motivations. We seek to understand which diagrams the current model composition techniques are able to work with. To date, little is known about to what extent the existing techniques support to matching, or even computing the similarity between specific-types of design models. To explore these questions, we have found 2581 papers and realized a comprehensive and thorough analysis in $40^{1}$. To carry out this in-depth investigation, we first had to define some search strategies for finding the papers.

TABLE I. RESEARCH QUESTIONS

\begin{tabular}{|c|c|}
\hline Question & Motivation \\
\hline $\begin{array}{l}\text { RQ1: What are the types } \\
\text { of diagrams addressed by } \\
\text { comparison techniques? }\end{array}$ & $\begin{array}{l}\text { Find out the types of diagrams that } \\
\text { comparision techniques support, } \\
\text { thereby revealing the diagrams that } \\
\text { have been considered important as } \\
\text { well as identify improvement points. }\end{array}$ \\
\hline $\begin{array}{l}\text { RQ2: What are the data } \\
\text { structures commonly used } \\
\text { in the comparison } \\
\text { algorithms? }\end{array}$ & $\begin{array}{l}\text { Pinpoint which data structures are } \\
\text { used in the comparison algorithms. }\end{array}$ \\
\hline $\begin{array}{l}\text { RQ3: What are the types } \\
\text { or categories used for } \\
\text { evaluating diagrams in } \\
\text { similarity approaches? }\end{array}$ & $\begin{array}{l}\text { Understand the different aspects in } \\
\text { required to evaluate diagrams. }\end{array}$ \\
\hline $\begin{array}{l}\text { RQ4: How fine-grained } \\
\text { are the comparison } \\
\text { techniques? }\end{array}$ & $\begin{array}{l}\text { Grasp how accurate and detailed are } \\
\text { the comparison techniques. }\end{array}$ \\
\hline $\begin{array}{l}\text { RQ5: What are the } \\
\text { comparison types? }\end{array}$ & $\begin{array}{l}\text { Explore if techniques are able to } \\
\text { compare using different comparison } \\
\text { strategies, thereby allowing to } \\
\text { improve the precision of the } \\
\text { similarity. }\end{array}$ \\
\hline $\begin{array}{l}\text { RQ6: Which empirical } \\
\text { strategies are used to } \\
\text { evaluate the comparison } \\
\text { techniques? }\end{array}$ & $\begin{array}{l}\text { Check the empirical strategies used } \\
\text { to evaluate the comparison } \\
\text { techniques. }\end{array}$ \\
\hline $\begin{array}{l}\text { RQ7: Is the approach } \\
\text { automatic, semi-automatic } \\
\text { or manual? }\end{array}$ & $\begin{array}{l}\text { Investigate the level of automation } \\
\text { used to compare models, thereby } \\
\text { revealing the degree of human } \\
\text { intervention required to compute the } \\
\text { similarity score between two models. }\end{array}$ \\
\hline
\end{tabular}

\section{B. Search Strategy}

To search for the studies, we have defined terms to form Search Strings for performing searches in the main digital libraries. These strings were formulated following well-known empirical guidelines, (e.g., [10][11][12][15]), and followed a

TABLE II. SEARCH STRING (SS)

\begin{tabular}{|l|c|}
\hline Major Terms & Synonym Terms \\
\hline Diagrams & design OR model OR design OR structure \\
\hline Comparison & match OR matching OR differencing OR similarity \\
\hline
\end{tabular}

five-step process to define the search terms as follows: (1) define the major keys; (2) identify alternative words,

\footnotetext{
$1_{\text {http://www.kleinnerfarias.com/publications/conference/seke2015 }}$
} 
synonyms or related terms to major keywords; (3) verify if the major keywords are contained in articles of the research category; (4) associate synonyms, alternative words or terms related to the main keywords with the Boolean "OR"; and (5) relate the major terms with Boolean "AND".

The major keywords are "Diagram" and "Comparison". Table II shows the synonyms and related words to major terms. We developed various combinations of Search Strings. However, we presented the substring that returned the most accurate results in search engines:

\section{((Diagram OR Design OR Model OR Structure) AND (comparison OR matching OR differencing OR match)))}

The search string above was used in the major search engines for academic studies of the Internet: IEEE Digital Library, Science Direct, Digital ACM Library, Scopus, Google Scholar and Springer Link.

\section{Selection: inclusion and exclusion criteria}

We have used the following criteria to include the primary studies. First, search was limited to studies published in electronic digital libraries from newspapers or journals, educational institutions, international conferences, Master and $\mathrm{PhD}$ thesis. Secondly, we only considered approaches written in English. Thirdly, there has been no restriction on the publication year of studies until November 2014. Finally, papers witch proposes model comparison.

For approach exclusion, we have applied the following criteria: (1) papers and studies witch not focus on model comparison; (2) duplicated studies returned by different search engines; and (3) papers and works that focus in low-level comparison (XML, source code and text).

\section{Extracted Data}

The following text describes the collected data we have extracted from articles to a spreadsheet and used it for summarizing the state-of-the-art model comparison techniques: (1) implicit data of inclusion and exclusion criteria: publication date, publication fora, and search engine; and (2) basic attributes of studies: main author and title; and finally (3) information related to research questions:

Diagrams (RQ1). The set of diagrams elicited from collected studies. They are accounted according these types of diagrams, including Component-and-Connector (CC), Generic (GD), Meta-Models (MM), Business Process Models (BPM), Use Case Diagram (UC), Class Diagram (CD), Sequence Diagram (SD), Activity Diagram (AD), Statechart Diagram (SCD), UML Profile (UP), and Any UML Diagram (AUD). Some diagrams are based in UML notation, but none of them was associated to a specific UML version.

Data Structures (RQ2). Basic data structures used by approaches and technologies.

Comparison aspects (RQ3). There is not a defined set of comparison aspects for model evaluation in the current literature. We have identified the following six comparison criteria in the works investigated: (1) structure, compare diagrams considering the modules and their relationships; (2) syntactic, compare taking into account the syntaxes of diagrams; (3) semantic, compare diagrams considering the meanings of the differences; (4) layout, the comparison approaches aim at view issues; (5) lexical, implement a namebased model comparison; and (6) multi-strategy, the approaches combine at least two comparison strategies to improve the comparison results.

Granularity levels (RQ4). Granularity refers to the unit of conflicts, e.g., attributes of the input models, and depends on the diagram used and criteria evaluated (item 3) e.g., layout aspects on UML class diagram own it is specific attributes. Users can set the level of granularity according the desired scalability and user's convenience [23]. We categorized the model comparison in tree levels of granularity: (1) coarsegrained, only one attribute is analyzed to compute the elements differentiation, e.g., the element names only; (2) partial, a set of attributes that is analyzed, i.e., more than one element; and (3) fine-grained, use all the possible attributes for execute the diagrams differentiation;

Comparison Type (RQ5). Comparison techniques can find the commonalities and differences between models using different strategies. We have identified two types of model comparison: (1) similarity, the mechanism's goal is to identify the similarity retuning values indicating how similar the elements between each other are; and (2) matching, the mechanisms return a set of matched elements.

Research method categories (RQ6). This is a question that provides a general view about the direction of the current studies, i.e., the kind of studies that academia have been producing. Given that there is a vast amount of works to be classified, we have used the categories proposed in [12] for classifying the selected papers: (1) evaluation research uses empirical strategies to evaluate proposed works; (2) solution proposal proposes a solution based on new or previous approaches; (3) validation research used for evaluating techniques, which have not been widely adopted in industry; (4) philosophical papers proposes new and revolutionary research to address some aspects of model comparison; and (5) opinion papers, studies that remain the discussion about the author's point of approaches arguing to resolve the tackled problem based in previously personal experiences.

Autonomous level (RQ7). In order to know the kind of automation support that algorithms provide to users, we have investigated the current works from tree perspectives: (1) automatic, it does not require any human interaction; and (2) semi-automatic, it requires users specifying configuration parameters before differentiation execution. Those approaches need user intervention for handling evaluation procedure, and (3) manual, a list with strategy steps or good practices for conducing the diagram comparison.

Technique description (RQ8). We have also observed how authors represent the comparison algorithms one. We generalized the following studies according the selected studies content: (1) pseudo-code, the approach shows the algorithm in a generic formalism, i.e. language-independent; (2) textual: authors to explain how the approach works by plain text; and (3) other, language-dependent and formalism representations (programming languages, modeling representations, etc.). 


\section{EXECUTION}

We have adopted the following four steps to select studies. The list below describes the sub-phases used to find studies, and Table III shows the results obtained in each sub-phase.

- $\quad$ Step 1. First results (SP1): find electronic papers using the substring, according Section III.B.

- $\quad$ Step 2. Duplicates Removed (SP2): remove repeated studies.

- $\quad$ Step 3. Pre Selection (SP3): remove papers that do not match in established requirements and research questions.

- $\quad$ Step 4. Selected Studies (SP4): we analyzed all selected studies in the previous step and applied the exclusion criteria aforemenioned.

TABLE III. STUDIES OBTAINED IN EACH STEP

\begin{tabular}{|c|c|c|c|c|c|c|c|}
\hline Steps & IEEE & Scopus & $\begin{array}{c}\text { Springer } \\
\text { Link }\end{array}$ & $\begin{array}{c}\text { Google } \\
\text { Schoolar }\end{array}$ & ACM & $\begin{array}{c}\text { Science } \\
\text { Direct }\end{array}$ & Total \\
\hline SP1 & 270 & 461 & 891 & 427 & 49 & 483 & 2581 \\
\hline SP2 & 268 & 321 & 787 & 392 & 45 & 476 & 2289 \\
\hline SP3 & 41 & 49 & 87 & 93 & 20 & 9 & 299 \\
\hline SP4 & 7 & 2 & 2 & 23 & 6 & 0 & 40 \\
\hline
\end{tabular}

\section{STUDY RESUlts}

This section presents the results for each research question as follows.

\section{A. Diagrams Category (RQ1)}

The majority of the works (14 approaches) focused on generic diagrams, i.e, those diagrams that are more abstract and consider similar attributes to compare the diagrams. Model comparison plays an important role inside MDE, where the capability in comparing many kinds of models are required. In addition, a recent study concluded most developers and large companies think UML complex leading the use of more abstract and alternative models [24]. This is the explanation more accepted about the high quantity of approaches focusing on resolving generic diagram comparison. On the other hand, the class diagram is the most common UML diagram investigated (12 approaches); similar to previous studies [25][26] in the field of software modeling highlighting UML class diagram as one of UML most used in practice. Although UML is considered de fact standard modeling language [26], we have observed the number of non-UML-based comparison techniques $(48 \%)$ ) outnumbers the UML-based ones $(53 \%)$.

\section{B. Data Structures Used (RQ2)}

Majority of the approaches (58\%) use graphs for model comparison. The tree data structure is the second most used $(14 \%)$ by authors. The minority $(3 \%)$ implemented semantic similarities, and other authors (25\%) utilize other simpler data structures.

\section{Comparison Aspects (RQ3)}

The results about comparison aspects revealed 21 approaches focusing in structural comparison aspects. After, 11 papers focused on Multi-Strategy comparison, i.e., comparison mechanisms using more than one strategy. Moreover, three papers evaluated the semantic aspects, and other 3 studies focused in lexical comparison, i.e., only evaluating the differences between words and one algorithm evaluated the layout characteristic, and one article focusing in syntactic aspect. The greater part of approaches evaluates one aspect, i.e., 29 approaches, and the remaining studies evaluated more than one aspect.

\section{Granularity (RQ4)}

Most approaches (29) proposed coarse-grained approach, i.e., they evaluate only one level of abstraction. Section V.A pointed that most approaches focused on generic diagrams. This leads model comparison algorithms considering main attributes for evaluating models. Other only three approaches executed a fine-grained evaluation, i.e., a detailed attributes coverage.

\section{E. Comparison Type (RQ5)}

We have classified the comparison mechanisms in two categories: (1) similarity, focusing on math similarity; and (2) matching, approaches performing a mapping of model elements. This definition is located in the Section III.D. The matching approach (28 of 40 studies) is the most used, followed by similarity approach (12 of 40 studies).

\section{F. Research Method (RQ6)}

The majority of appraches $(77,5 \%, 31$ of 40 papers) are Proposal of Solution. Evaluation Research and Philosophical Papers has the same quantity $(10 \%, 4$ of 40 papers) and, the resting approaches $(2,5 \%, 1$ of 40 papers) rely on practical experience to develop a comparison approach. There are two main aspects to consider in this research question: (1) there are new emerging approaches for model comparison; and (2) the approaches do not complement each other. The results show that the literature has recurrently proposed and discussed new comparison techniques. During the whole selection steps, we did not find any opinion paper about model comparison.

\section{G. Autonomously Level (RQ7)}

Most algorithms conduct the model comparison process autonomously $(67,5 \%, 27$ of 40 papers), followed by semiautomatic process $(30 \%, 12$ of 40 papers) where users must set some adjustments during the comparison and, only one approach $(2,5 \%, 1$ of 40 studies) discusses how persons manually match UML diagrams. From the results is possible to perceive a strong tendency for producing automatic approaches. We understand the focus in the process of diagram comparison is to avoid users wasting time with specific configurations.

\section{COMPLEMENTARY RESUlTS}

Table IV shows a list that contains a rank of those publication fora that contains more papers focusing on comparison of diagrams. Table represents $27.5 \%$ of all papers analyzed (11 of 40 articles). Conferences/Journals with one article did not appeared in this Table. Figure 1 presents that the frequency of publications was higher in 2008 and 2011. This period was responsible for producing 19 studies, i.e., a higher quantity production than other periods from 2003 to 2007, and from 2012 until 2014. 
TABLE IV. QUANTITY OF PAPERS PER EVENT/JOURNAL

\begin{tabular}{|l|c|c|}
\hline \multicolumn{1}{|c|}{ Publication Place } & $\begin{array}{c}\text { Quantity of } \\
\text { approaches }\end{array}$ & Percentage \\
\hline $\begin{array}{l}\text { IEEE/ACM International } \\
\text { Conference on Automated } \\
\text { Software Engineering (ASE) }\end{array}$ & 4 & $10 \%$ \\
\hline $\begin{array}{l}\text { IEEE Transactions on software } \\
\text { Engineering }\end{array}$ & 3 & $8 \%$ \\
\hline $\begin{array}{l}\text { European Software Engineering } \\
\text { Conference and the ACM } \\
\text { SIGSOFT Symposium on The } \\
\text { Foundations of Software } \\
\text { Engineering (ESEC/FSE) }\end{array}$ & 2 & $5 \%$ \\
\hline $\begin{array}{l}\text { International Conference on } \\
\text { Software Maintenance (ICSM) }\end{array}$ & 2 & $5 \%$ \\
\hline
\end{tabular}

The production was the most unproductive in 2009 (just 1 article produced). In a general overview over the chart is possible to perceive a frequent times of rise and falls in publication numbers. Moreover, the average of produced studies is low (by about 3,33 studies per year).

Figure 1. Publications by year

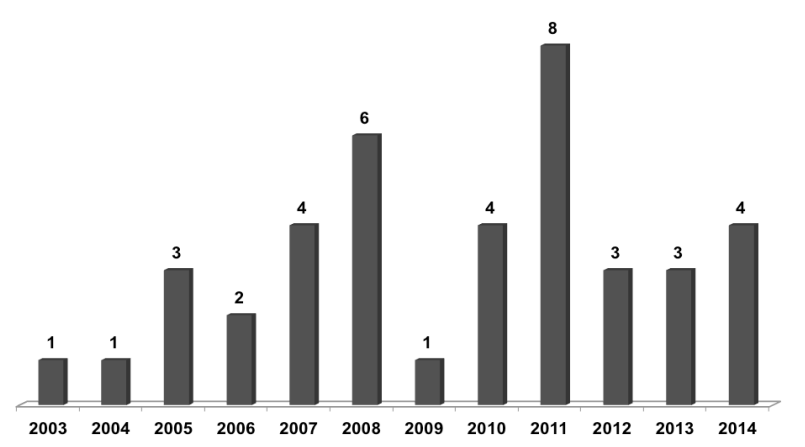

Table $\mathrm{V}$ shows a rank of all authors according their productivity in relation to matching and similarity approaches publications. The number of papers was accounted taking in consideration the author's first name in studies. Zhenchang Xing is the author that most produced comparison techniques ( $8 \%, 3$ from 40 studies). After six authors produced two papers, and others authors produced each one approach.

TABLE V. Authors Publication QUANTITY RANKING

\begin{tabular}{|l|c|c|}
\hline \multicolumn{1}{|c|}{ Author } & $\begin{array}{c}\text { Quantity of } \\
\text { Approaches }\end{array}$ & Percentage \\
\hline Zhenchang Xing & 3 & $8 \%$ \\
\hline Christian Gerth & 2 & $5 \%$ \\
\hline Hamza Onoruoiza Salami & 2 & $5 \%$ \\
\hline Kleinner Oliveira & 2 & $5 \%$ \\
\hline Mark van den Brand & 2 & $5 \%$ \\
\hline Segla Kpodjedo & 2 & $5 \%$ \\
\hline Shiva Nejati & 2 & $5 \%$ \\
\hline
\end{tabular}

The following discussion describe the analysis of the results (Figure 2) for the combined research questions illustrated in a bubble plots. This method gives a map and provides a general overview of what academia published. Combining the results of RQ1, RQ6, and year, we obtained the mapping of the evolution through years of the number of type diagrams and the research methods used. The results show that the proposal of solution is the research type that have been more adopted by academia to present comparison approaches over the last 11 years. In 2008, all 6 papers were proposal of solution, where 3 focused the comparison approach on class diagrams and respectively one approach for generic, meta-model and UML profiles. Generic diagram received more attention by three years in academia. Firstly, in 2007, two proposals were published concerning generic comparison of diagrams. This happened after by about six years from the Model-Driven Architecture (MDA) has been formally proposed, and according our results, after four years, the beginning of the model comparison studies in the academia. Second, in 2010, all four proposals of solutions were concerned in generic diagram comparison; a year before (2009) only one study was published with focus on class diagrams, i.e., the most unproductive year. Finally, in 2011, generic digram was the center of attention by the second year consecutive.

\section{THREATS TO VALIDITY}

We follow the systematic mapping study methodology for the execution of this research. This method provides protocols to extract data in order to guarantee detailed results of the state of the art. For this we defined the search strings and research questions. However, some factors may threat the validity of the study: (1) difficulty to relate all works to the topic due the constant changes in publications; and (2) the conduction of data extraction of the papers, such as (1) the search string we used has the main terms such as "model" and "matching". However, "matching" and its synonyms (comparison, similarity, etc.) are generic and this string retrieved broad results; (2) the inclusion of thesis and dissertations published on-line that are not peer revied and, (3) the limitation to the main six search engines defined in the SMS planning.

\section{CONCLUSION}

This paper identified and classified publication fora, and performed thematic analysis of the existing literature in model comparison, thereby providing an in-depth understanding about the model comparison area. In addition, it addressed this gap by describing and pinpointing in which field and for which research topics a shortage of publications still exits.

We have observed that the most studies have concentrated more effort on producing generic comparison techniques, rather than on providing specific ones, e.g., techniques for comparing UML models. This can be explained by three reasons. First, there is not a widely-adopted modeling language in industry. Second, given the wide variations of modelling notations and diagrams types, it would be challenging to provide an approach that can have a broad adoption. Third, model comparison is not a trivial task to deal with. Rather, it may be still characterized a time-consuming and error-prone task. Finally, we also hope that this work represents a first step in a more ambitious agenda on providing a better support researchers and practitioners to compare models. In addition, we hope that the issues outlined throughout the paper may encourage other researchers to extend our study. 


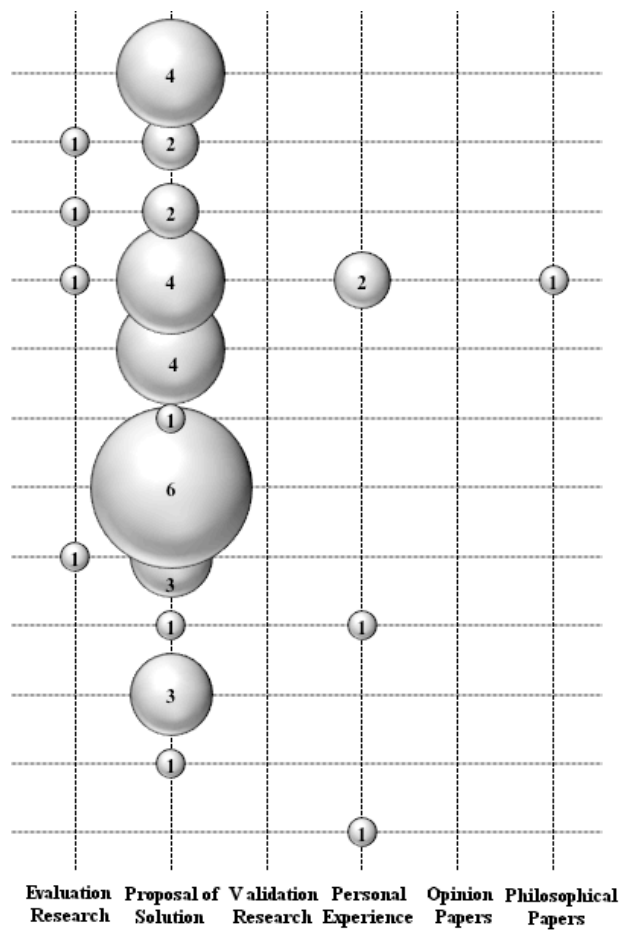

ACKNOWLEDGMENT

This work was funded by Universal project - CNPq (grant number 480468/2013-3).

\section{REFERENCES}

[1] S. Kent, "Model-driven engineering," In: 3rd Int. Conf. on Integrated Formal Methods (IFM '02), pages 286-298, 2002.

[2] A. Sarma, D. Redmiles, A. Van der Hoek, "Palantir: early detection of development conflicts arising from parallel code changes," Software Engineering, IEEE Transactions on , vol.38, no.4, pp.889,908, July-Aug. 2012.

[3] Wieland et al. "Turning conflicts into collaboration," Computer Supported Cooperative Work, pages 181-240, 2013.

[4] IBM, IBM Rational Software Architecture. http://www.ibm.com/developerworks/downloads/r/architect/index.html, accessed in 2015.

[5] Epsilon, https://www.eclipse.org/epsilon/, accessed in 2015.

[6] J. Whittle, P. Jayaraman, Synthesizing hierarchical state machines from expressive scenario descriptions, ACM Trans. Softw. Eng. Methodol, vol. 19, no. 8, February 2010.

[7] K. Voigt, "Structural graph-based metamodel matching," $\mathrm{PhD}$ thesis, University of Desden, 2011.

[8] S. Abbas, H. Seba, "A module-based approach for structural matching of process models," 5th Int. Conf. on Service-Oriented Computing and Applications, pages 17-19, 2012.

[9] K. Petersen, R. Feldt, S. Mujtaba, M. Mattsson, "Systematic mapping studies in software engineering," 12th Int. Conf. on Evaluation and Assessment in Software Engineering, UK, pp. 68-77, 2008.

[10] D. Budgen et al., "Using mapping studies in software engineering," Proceedings of PPIG, vol. 8, pp. 195-204, 2008.

[11] B. Kitchenham, P. Brereton, D. Budgen, "The educational value of mapping studies of software engineering literature," 32nd Int. Conf. on Software Engineering, vol. 1, New York, NY, USA, pp. 589-598, 2010.

[12] B. Kitchenham, D. Budgen, O. Brereton, "Using mapping studies as the basis for further research - A participant-observer case study," Inf. Softw. Technology, pp. 638-651, 2011.
[13] D. Torre, Y. Labiche, M. Genero, "UML consistency rules: a systematic mapping study," 18th Int. Conf. on Evaluation and Assessment in Software Engineering, 2014.

[14] N. Asoudeh, Y. Labiche, "Requirement-based software testing with the UML: a systematic mapping study," $7^{\text {th }}$ Int. Conf. on Software Engineering Advances, 2012.

[15] Wohlin et. al., "Experimentation in Software Engineering", Springer, Heidelberg, Berling, Germany, 2012.

[16] OMG, UML metamodel: superstructure specification, http://www.omg.org/spec/UML/2.4.1/Superstructure/PDF, 2015.

[17] D. Kolovos, D. Ruscio, A. Pierantonio, R. Paige, "Different models for model matching: an analysis of approaches to support model differencing", Workshop on Comparison and Versioning of Software Models(CVSM '09), pages 1-6, 2007.

[18] M. Stephan, J. Cordy, "A survey of model comparison approaches and applications," in International Conference on Model-Driven Engineering and Software Development (MODELSWARD), pp.265-277, 2013.

[19] K. Altmanninger, S. Martina, M. Wimmer, "A survey on model versioning approaches," Int. Journal of Web Information Systems, pages 271-304, 2009

[20] M. Alanen, I. Porres, "Difference and union of models," UML conference, pages 2-17, 2003.

[21] H. Salami, M. Ahmed, "UML artifacts reuse: state of the art," 2014.

[22] P. Selonen, "A review of UML model comparison approaches," Nordic Workshop on Model Driven Engineering, 2007.

[23] B. Bruegge, A. de Lucia, F. Fasano, G. Tortora, "Supporting distributed software development with fine-grained artefact management," $6^{\text {th }}$ Int Conf. on Global Software Engineering, pages 213-222, 2006.

[24] M. Petre, "UML in practice," International Conference on Software Engineering, Piscataway, NJ, USA, pages 722-731, 2013.

[25] A. Nugroho, M. Chaudron. "A survey into the rigor of UML use and its perceived impact on quality and productivity," In Proceedings of the Second ACM-IEEE international symposium on Empirical software engineering and measurement (ESEM '08). ACM, New York, pp. 90-99, 2008

[26] M. Chaudron, W. Heijstek, and A. Nugroho. "How effective is UML modeling ?" Software System Modeling. Vol. 11, no. 4, pp. 571-580, 2012 . 